\title{
UK government collaborations to manage threats to animal and human health
}

\author{
The chief veterinary and chief medical officers are working closely together on bird \\ flu and other zoonoses
}

$\mathrm{T}$ The confluence of human and animal health has, once again, been brought under the spotlight by the current epidemic of avian influenza (H5N1 virus) affecting poultry and humans across South East Asia and by its recent spread into Europe. Understanding and developing the multiple links between these two fields is essential for establishing effective surveillance, preparedness, and response strategies and for developing appropriate, government-wide mechanisms for risk assessment and management.

As chief medical officer (CMO) and chief veterinary officer (CVO), we have a shared responsibility to communicate with both professional and public audiences. We hope that this article will support other communications in illustrating the breadth and depth of the strategic collaboration between our two professions within the United Kingdom government. In addition, we hope that the article will prompt deeper inquiry by the sister professions into increasing their participation in these important relationships.

We have worked together-and with many colleagues-on influenza and other zoonotic infections that threaten human health in a variety of ways. As heads of our professions, we recognise the fundamental differences between the roles and responsibilities of vets and doctors. Our collaboration on influenza reminds us, however, of the importance of encouraging dialogue between our respective professions in order to strengthen the capacity of veterinary and public health both nationally and locally.

The avian influenza virus $\mathrm{H} 5 \mathrm{~N} 1$ has the potential to bring together human illness (normal seasonal flu) and an animal disease (bird flu), causing illness in some people in affected countries (though bird flu is not, fortunately, in the UK at present) where there has been close contact between people and infected birds. In addition, there is the separate possibility, at some time in the future, of a human influenza pandemic. This complex scenario creates an arena in which the medical and veterinary disciplines bring distinct, but interrelated, professional skills together to help solve any problems associated with the interaction between people and animals.

The current outbreak of avian influenza caused by virus $\mathrm{H} 5 \mathrm{~N} 1$ is not the first zoonotic infection to show the importance of having a close working relationship between the chief veterinary and chief medical officers. The emergence of variant Creutzfeldt-Jakob disease in humans and the possibility that this might be linked to the epidemic of bovine spongiform encephalopathy (BSE) in cattle highlighted the need to collaborate in assessing the potential threats to human health posed by animal diseases. The 2001 epidemic of foot and mouth disease (FMD) and the challenge it posed for the disposal of infected animal carcasses reminded us of the need to consider the wider potential impacts of animal diseases even when the disease itself seemed to have little public health importance for humans. Furthermore, the

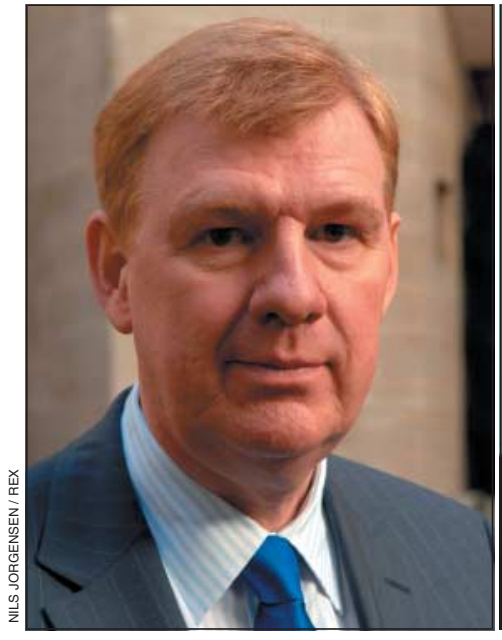

Liam Donaldson, chief medical officer

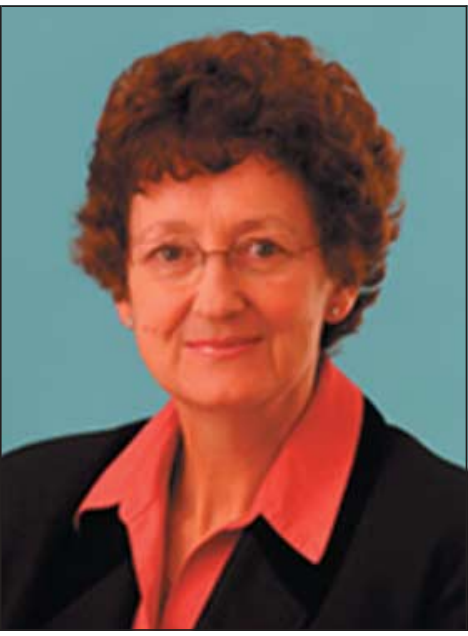

Debby Reynolds, chief veterinary officer emergence of severe acute respiratory syndrome (SARS) clearly showed the need to collaborate with our chief medical and chief veterinary counterparts in European Union member states and worldwide via the World Organisation for Animal Health (OIE), the World Health Organization, and the various expert advisory groups.

Such collaboration is essential for several reasons. Firstly, cooperative relationships are vital to the development by governments of mechanisms for strategic risk assessment and risk management. This has been illustrated, for example, by the wide economic impacts of BSE and FMD in the UK and of H5N1 globally. This is why the CVO and CMO have a shared responsibility to report occurrences of new and emerging zoonoses and to advise ministers on appropriate risk management measures. At the heart of this shared responsibility is a commitment by both the $\mathrm{CVO}$ and CMO to rapid and open sharing of information, transparency in the risk assessment process, and communication of those risks to stakeholders.

Secondly, collaboration is vital to the development of a robust and responsive infrastructure for animal and human health surveillance. In response to recommendations made by the CMO in his 2002 annual report, the establishment, in 2003, of the National Expert Panel on New and Emerging Infections facilitated the integration of data gathering for animal and human health surveillance and strengthened the assessment of potential threats to health from new and emerging diseases, particularly zoonoses. This has been supported by the foundation of the Health Protection Agency, tasked with aligning more closely information on human and animal health surveillance.

The CVO's 2002 review of veterinary surveillance in England and Wales concluded that such surveillance 
needed to be more risk based and that risk management decision making should be more open and transparent, with clearer and wider communication of the surveillance data. The Department for Environment, Food, and Rural Affairs (Defra) launched its new Veterinary Surveillance Strategy in 2003, putting at its core the protection of public health in relation to food and animal diseases transmissible to people.

Thirdly, the integration of human and animal health is vital for the analysis of data on human and animal infections and the sharing of this information with all those who have a role in managing risks and threats to public health. This is reflected in the existence of various joint expert advisory committees, official working groups, stakeholder groups, and international partnerships, which often consider specific issues contemporaneously (see bmj.com for detailed examples).

There is a risk, of course, that this large number of partnerships could, if not working cooperatively, hinder rather than help the implementation of an effective response to a new zoonotic infection. It is essential, therefore, that when a new, potentially zoonotic, and acute risk emerges these links can be "fast tracked," and mechanisms are in place that allow rapid assessment of the risks by using agreed methods for consistency, particularly when the risks to health are unclear.

A recent example of how the various groups work together is the development of health protection measures after the emergence of avian influenza. Independent advice was sought from the expert Joint Committee on Vaccination and Immunisation on the use of seasonal flu vaccine for poultry workers and others at risk groups and from the Advisory Committee on
Dangerous Pathogens on the wider risks associated with exposure of the public or workers to $\mathrm{H} 5 \mathrm{~N} 1$ and other influenza viruses. In this context, staff embraced all sectors from agriculture to the NHS. The advice received has been made public and has been used to produce specific guidance for affected industry groups.

Examples of other collaborative working on zoonotic diseases, coordinated through the UK Zoonoses Group, are the development of common standard operating procedures by the veterinary and public health Laboratory Test Group for the isolation, identification, and typing of bacterial, viral, and parasitic organisms and for antibiotic sensitivity testing, and the development of shared databases incorporating phenotypic and molecular information from human and animal sources. Other projects integrating human and animal health, such as the harmonisation of procedures for the surveillance of animal and human infectious disease and the cross training of veterinary and public health staff in new techniques for handling new or unusual zoonotic pathogens, increase our ability to scale up laboratory diagnostics should the need arise.

These diverse and cooperative ways of working across the animal and human health spectrum allow us to identify, and develop measures to better manage, animal or human disease threats that may arise either within the UK or globally.

Debby Reynolds chief veterinary officer

Department for Environment, Food, and Rural Affairs, Nobel House, London SW1P 3JR (cvo-uk@defra.gsi.gov.uk)

Liam Donaldson chief medical officer

Department of Health, Richmond House, London SW1A 2NL

Competing interests: None declared.

\section{Foodborne zoonoses}

\section{Food poisoning can be serious, and doctors and vets have key roles in tackling it}

$\mathrm{F}$ oodborne disease (food poisoning) tends to be regarded as a comedy illness-not pleasant to have, or talk about, but little more than an inconvenience. Yet trivialising foodborne disease ignores the size of the illness burden: estimates vary from 76 million cases of foodborne disease annually in the United States ${ }^{1}$ to 5.4 million in Australia ${ }^{2}$ and 1.3 million in England and Wales. ${ }^{3}$ Three of the major pathogens-Campylobacter spp, Salmonella spp, and Shiga toxin producing Escherichia coli O157 (STEC O157)-are zoonoses (that is, transmitted from vertebrate animals to humans). As well as causing acute symptoms including diarrhoea and vomiting, infection can have long term implications.

Campylobacter is the principal bacterial cause of gastroenteritis in the developed world. The World Health Organization estimates that about $1 \%$ of the population of Europe will be infected with Campylobacter spp each year. In England and Wales about 45000 acute cases are diagnosed annually. Infection is associated with development of Guillain-Barré syn- in polio-free regions in the world. ${ }^{4}$ The risk of developing it after campylobacter infection is about 1 in 1000 (the risk rising to around 1 in 160 for infections with certain serotypes). ${ }^{5}$ Both campylobacter and salmonella infections can result in reactive arthritis, although the precise mechanisms are still unclear, and infection with STEC O157 is a leading cause of haemolytic uraemic syndrome, the most common preventable trigger for acute renal failure in children. ${ }^{6}$

Clinical management of patients with acute symptoms of foodborne disease in primary care is generally the same regardless of aetiology (rest and rehydration), and treatment with antimicrobials is rarely indicated for uncomplicated diarrhoea. For example, there is no evidence that antibiotic treatment of uncomplicated salmonella diarrhoea in otherwise healthy children and adults is beneficial, and it may even prolong salmonella carriage. ${ }^{7}$ Antimicrobial treatment in cases of STEC O157 infection might do positive harm, potentially precipitating the onset of haemolytic uraemic syndrome. ${ }^{8}$ Limiting inappropriate 